around these nodules reiterating the importance of prompt initiation of chemotherapy in these patients [3]. Our patient achieved full neurological recovery despite extensive intracranial haemorrhage following prompt initiation of chemotherapy.

\section{Conclusion}

Our case report expands on the neuroimaging findings of a rare distinctive diffuse intracranial haemorrhage associated with ALL hyperleukocytosis. Despite the extensive nature of the haemorrhagic lesions, our report highlights that prompt supportive and cytoreductive treatment can offer potentially full neurological recovery in these group of patients.

\section{Conflict of interest/disclosures}

The authors declare that they have no financial or other conflict of interest in relation to this research and its publication.

\section{References}

[1] Steliarova-Foucher E, Columbet M, Ries LAG, et al. International incidence of childhood cancer, 2001-10: a population-based registry study. Lancet Oncol 2017; 18:719-31.

[2] Fritz RD, Forkner CE, Freireich RJ, et al. The association of fatal intracranial hemorrhage and "blastic crisis" in patients with acute leukaemia. N Eng J Med 1959;261:59-64.

[3] Freireich EJ, Thomas LB, Frei E, et al. A distinctive type of intracerebral hemorrhage associated with "blastic crisis: in patients with leukemia. Cancer 1960;13:146-54.

[4] Koenig MK, Sitton CW, Wang M, et al. Central nervous system complications of blastic hyperleukocytosis in childhood acute lymphoblastic leukemia: diagnosis and prognostic implications. J Child Neurol 2008;23(11):1347-52.

[5] Ruggiero A, Attina G, Piastra M, et al. Severe hyperleukocytosis and multifocal intracranial haemorrhage: not always a fatal outcome. Int J Hematol 2009;90:87-90.

[6] Shiber JR, Fines RE. Cerebral hemorrhage due to hyperleukocytosis. J Emerg Med 2011;40(6):674-7.

[7] Munoz J, Donthireddy V. CNS hyperleukocytosis. Blood 2012;119:5953.

\title{
Pharmacoresistant seizures in neurofibromatosis type 1 related to hippocampal sclerosis: Three case presentation and review
}

\author{
Demet Illhan Algın ${ }^{\mathrm{a}, *}$, F. Irsel Tezer ${ }^{\mathrm{a}}$, Kader K. Oguz ${ }^{\mathrm{b}}$, Burcak Bilginer ${ }^{\mathrm{c}}$, Figen Soylemezoglu ${ }^{\mathrm{d}}$, Serap Saygi ${ }^{\mathrm{a}}$ \\ ${ }^{a}$ Department of Neurology, School of Medicine, Hacettepe University Hospitals, Ankara, Turkey \\ ${ }^{\mathrm{b}}$ Department of Radiology, School of Medicine, Hacettepe University Hospitals, Ankara, Turkey \\ ${ }^{\mathrm{c}}$ Department of Neurosurgery, School of Medicine, Hacettepe University Hospitals, Ankara, Turkey \\ ${ }^{\mathrm{d}}$ Department of Pathology, School of Medicine, Hacettepe University Hospitals, Ankara, Turkey
}

\section{A R T I C L E I N F O}

\section{Article history:}

Received 25 February 2019

Accepted 25 March 2019

\section{Keywords:}

Neurofibromatosis

Hippocampal sclerosis

Intractable seizures epilepsy surgery

\begin{abstract}
A B S T R A C T
Neurofibromatosis type 1 (NF1) is an autosomal dominantly inherited disorder, with an estimated prevalence of 1 in 3000-4000 people. Seizures occur $4-7 \%$ of individuals with NF1, mostly due to associated brain tumors or cortical malformations. Hippocampal sclerosis (HS) in the patients with NF1 has been reported very rarely and only 15 patients were found in review of English literature. We presented here 3 additional patients with NF1 and intractable seizures due to hippocampal sclerosis; in whom one of them underwent epilepsy surgery and he is seizure free for 5 years after right temporal lobectomy.
\end{abstract}

(c) 2019 Elsevier Ltd. All rights reserved.

\section{Introduction}

Neurofibromatosis type 1 (NF1) is an autosomal dominant disorder characterised by multiple "cafe-au-lait" macules, neurofibromas, intertriginous freckling and Lisch nodules. Previous reports have estimated that seizures occur in approximately $4-7 \%$ of individuals with NF1 [1-6]. It was found that epileptic seizures are related to various types of intracranial lesions in NF1 patients. Associated malformation types have been described as hemimegalencephaly, cerebellar leptomeningeal heterotopias, transmantle cortical dysplasia, a periventricular band of heterotopic grey matter, pachygyria, occipital encephalocele, and unilateral as well as bilateral polymicrogyria. In patients with NF1, seizures are often focal, and focal abnormalities are seen in EEGs. Seizure control is difficult with monotherapy, and drug resistant epilepsy rate is relatively high [7]. HS has been reported very rare in the patients with

* Corresponding author at: Dept. Of Neurology at Eskisehir Osmangazi University Hospital, Eskisehir, Turkey.

E-mail addresses: ilhandemet@gmail.com (D.Ilhan Algın), burcak@bilginer.org (B. Bilginer), ssaygi@hacettepe.edu.tr (S. Saygi).
NF1 $[8,9]$. Here we report additional 3 cases with hippocampal sclerosis and NF1 after detailed investigation, in whom one of them underwent temporal lobectomy and he is seizure free for 5 years after surgery.

\subsection{Case 1}

A sixteen year old right-handed male patient has epilepsy history since the age of five and admit to our Epilepsy Clinic because of increased seizure frequency such as once a week or one-four times a day. Ictal behavioral characteristics were described in the form of bilateral automatism (hand and oral automatism), loss of consciousness and postictal confusion. Seizures had been persisted despite several drugs such as valproic acid, levetiracetam and pregabalin. He was on carbamazepine and pregabalin combined treatment at the admission. On the physical examination, there were extensive cafe au lait stains in the whole body, axillary freckles more than 5 and greater than $1 \mathrm{~cm}$ on the flexor and extensor side of right arm, abdomen, back and thigh and soft nodules on the back and right thigh lateral as well as lisch nodules on iris on the ophthalmic examination. According to family history, his grandmother, 


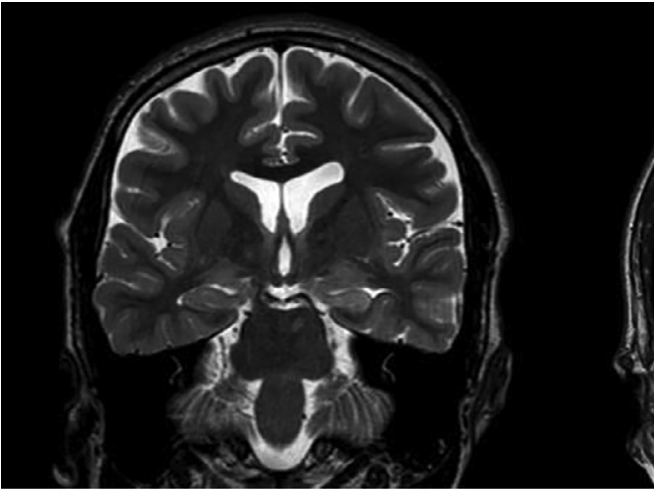

A

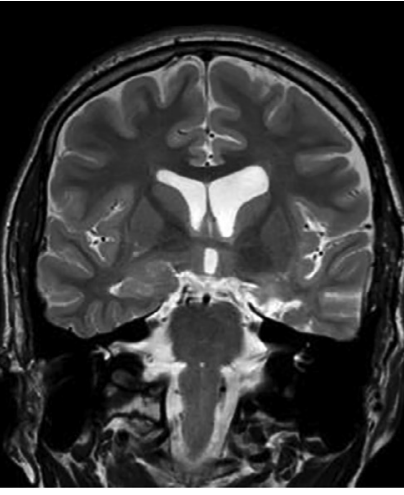

B

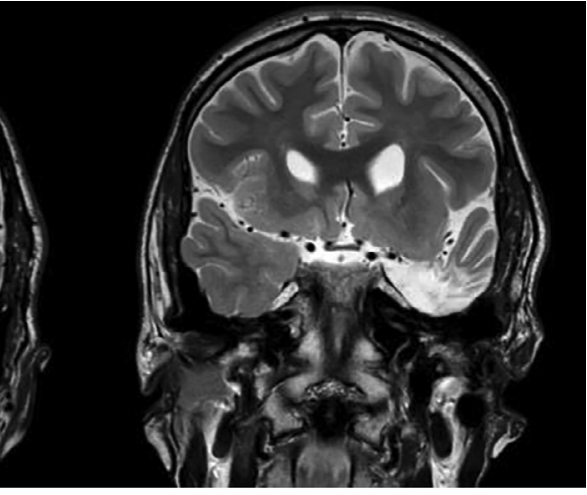

$\mathrm{C}$

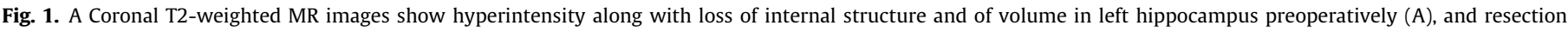
cavities filled with CSF due to left temporal lobectomy and hippocampectomy (B, C).

mother and uncle had the same signs. Two seizures were recorded on video EEG monitoring during 6-day hospitalisation. Focal seizures were consisted of oral and bilateral hand automatism, mild left deviation of the head and loss of. Ictal EEG showed rhythmic sharp slow wave activity on the left temporal region. Cranial magnetic resonance imaging (MRI) revealed few hyperintense unidentified bright objects (UBOs) on T2-weighted images in the pons, thickening and mild T2 hyperintensity on bilateral amygdala, and T2 hyperintensity accompanying loss of internal structure with a bit volume loss suggesting left hippocampal sclerosis (Fig. 1). On functional cerebral MRI, it was noticed that the right hemisphere was dominant by the blood-oxygen-level-dependent (BOLD). In our weekly multidisciplinary epilepsy meeting, left temporal lobectomy decision was made. He underwent left temporal lobectomy with hippocampectomy and amygdalectomy. Pathological investigation showed type I HS (2013 ILAE classification based on the pathology HS). He is seizure free for 5 years after surgery.

\subsection{Case 2}

A 34-year-old right-handed male patient with a previously known diagnosis of neurofibromatosis admitted to our clinic for intractable focal seizures. Age at onset for seizures was 9. Focal seizures with impairment of awareness and staring following aura (rising epigastric sensation) were described. Seizures rarely could evolve bilateral generalized convulsions. The frequency of seizures were 3-4 times a day or 2-3 times a month despite the lamotrigine, levetiracetam and carbamazepine combined therapy with high dosages. Physical examination revealed an extensive cafe au lait stains on the whole body, axillary freckles greater than $1 \mathrm{~cm}$ on the abdomen, thigh and back and soft nodules on the back and lower extremity. Family history was positive; his mother had the same complaints. He underwent five-day video-EEG monitoring. On interictal electroencephalography (EEG), sharp wave and spike-like epileptiform discharges apparent during the sleep were observed in the left temporal region. During the stay, two focal seizures with the loss of consciousness were recorded. Clinically, eyes deviated upward, there were dystonic posture on the right leg, and oral automatism and the patient wiped his nose with his left hand. At the same time, he could not answer the questions. Electrophysiologically, attenuation following the left temporal spike activity and subsequently left temporal rhythmic theta discharge spreading to the right side for 2 min were observed. Cranial MRI revealed there was T2 hyperintensity and loss of internal structure in the left hippocampus on T2-weighted images (Fig. 2). A left hippocam-

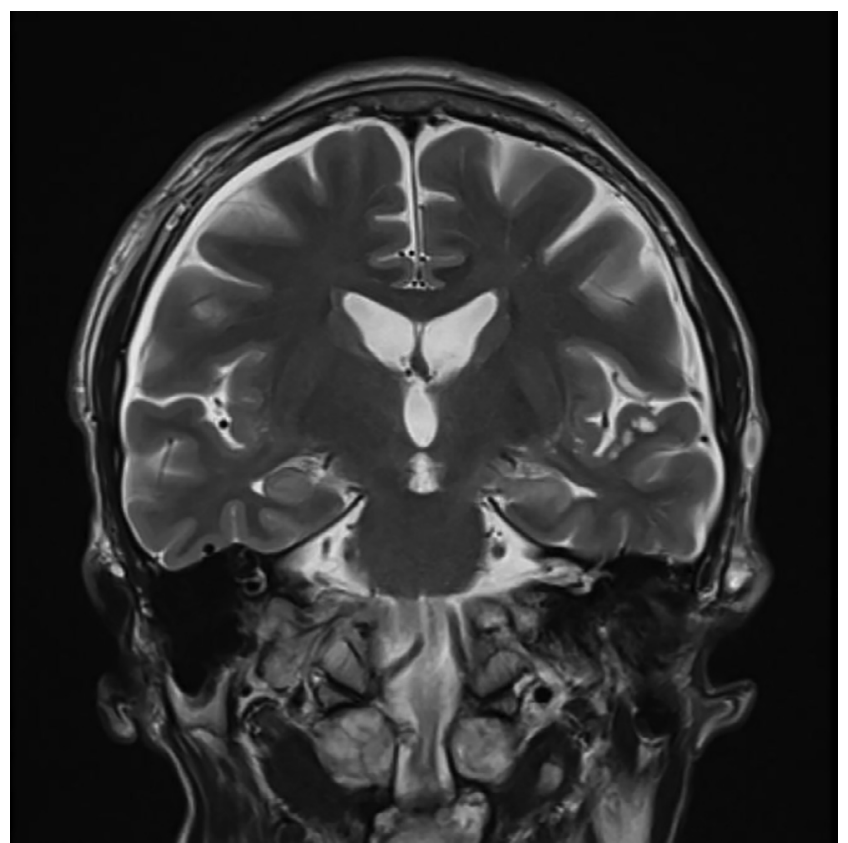

Fig. 2. Coronal T2-weighted MR image demonstrates hyperintensity and loss of internal structure in the left hippocampus.

pectomy was scheduled based on ictal EEG and cerebral MRI findings.

\subsection{Case 3}

A 54-year-old right-handed female patient with a previously known diagnosis of neurofibromatosis and epilepsy applied due to increased frequency of seizure. Her first seizure was started when she was 18 years old. Previously, the patient had seizures during the sleep once a month or every two months, but she had three seizures during the last month. Seizures were as follows; stare absently, automatism such as picking at clothes, subsequently often crying, unconscious behaviours (singing etc.) and often falling forward and sometimes falling back while standing. The previously used and ineffective antiepileptic drugs were topiramate, lamotrigine, barbexaclone, phenytoin and levetiracetam. Family history was unremarkable. Her medical history indicated depression and hypothyroidism. Physical examination revealed an extensive cafe au lait stains on the whole body, multi- 
ple neurofibromas and scoliosis. On neurological examination, the only postural tremor was noticed. On interictal electroencephalography (EEG), independent sharp wave or spike discharges were rarely seen in the right temporal and left frontotemporal areas. Four epileptic seizures were recorded during the long term video-EEG monitoring, the patient stared, and tried to get out of bed. Also, bilateral hand automatism and unilateral blinking were observed without awareness. On ictal EEGs, rhythmic delta activity appeared on the left temporal region, and it was propagated to the right temporal area within 5-6 s. Cranial MRI revealed decreased volume, loss of internal structure and T2 hyperintensity in the right hippocampus consistent with hippocampal sclerosis (Fig. 3). EEG recordings with intracranial electrodes have been planned due to discordance of MRI and EEG findings but the patient refused fur-

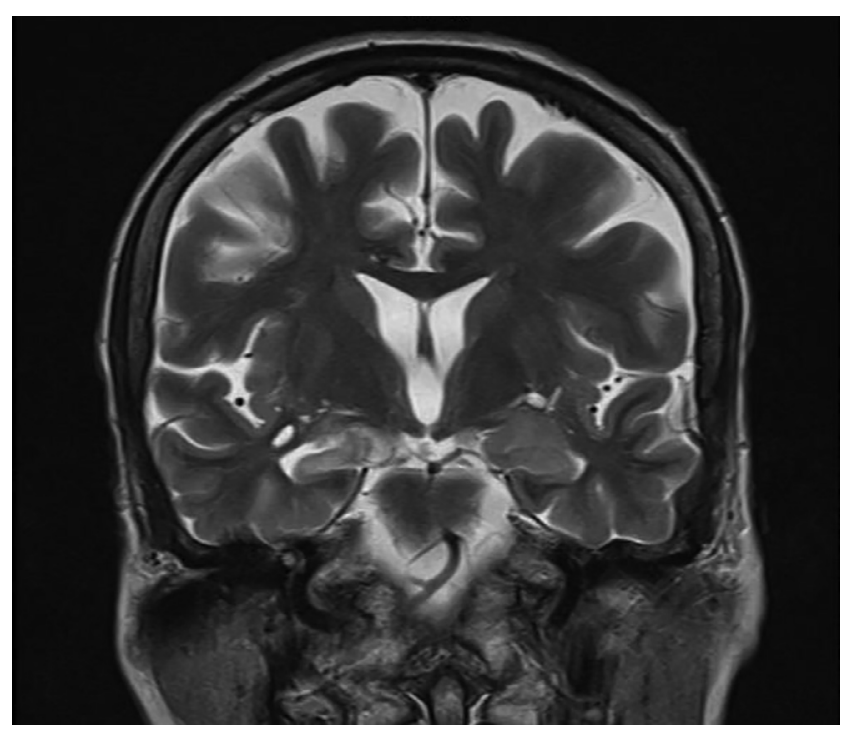

Fig. 3. Coronal T2-weighted MR image shows $\mathrm{T} 2$ hyperintensity along with atrophy in the right hippocampus suggestive of hippocampal sclerosis. ther investigation and epilepsy surgery. Her seizures still persist during ten year follow up after monitoring as an once a month.

\section{Discussion}

Neurofibromatosis type 1 (NF1) is an autosomal dominantly inherited disorder, with an estimated prevalence of 1 in 30004000 people. The NF1 gene provides instructions for making a protein called neurofibromin [10]. Neurofibromin acts as a tumour suppressor protein. This protein is produced in many types of cells, including nerve cells and specialised cells called oligodendrocytes and Schwann cells that surround nerves. Positive family history is noted in about $50 \%$ of cases [11]. Epilepsy is seen in $4-7 \%$ of patients with neurofibromatosis. This rate is higher than the reported rate as $1-2 \%$ for the general population. Seizures in NF1 tend to have focal onset and commonly generalize secondarily [12]. It is thought that seizures in NF1 arise from the numerous focal lesions that comprise the disorder, namely, tumors and malformations of cortical development. Thus, seizure occurrence requires neuroimaging, even if previous neuroimaging was normal. The relationship of UBOs to seizures is controversial, but most studies have concluded that UBOs are not associated with seizures [13,14] These are seen in about $64 \%$ of individuals, located in the brainstem, thalamus, basal ganglia and cerebellum [15].

In fifteen patients with NF1 and epilepsy, brain MRI has been able to show also hippocampal sclerosis in the review of English published reports so far. Of them, 9 were women, and 6 were men. Right HS was found in 10, left HS was found in 6 and bilateral HS was seen in 1 patient. Only one of the 15 patients had normal cerebral MRI (Table 1).

Vivarelli et al. have been identified 9 patients with NF1 and brain lesions. There was a cerebral tumour in 5 , a cortical malformation in 3 and HS in 1 patient. The authors reported that patient with HS responded to the treatment [16].

Jang et al. reported a patient diagnosed with resistant epilepsy associated with NF1 and right HS. They noted that the patient became seizure-free after surgical treatment [17]. In a study by

Table 1

Clinical characteristics of patients with NF1 and HS.

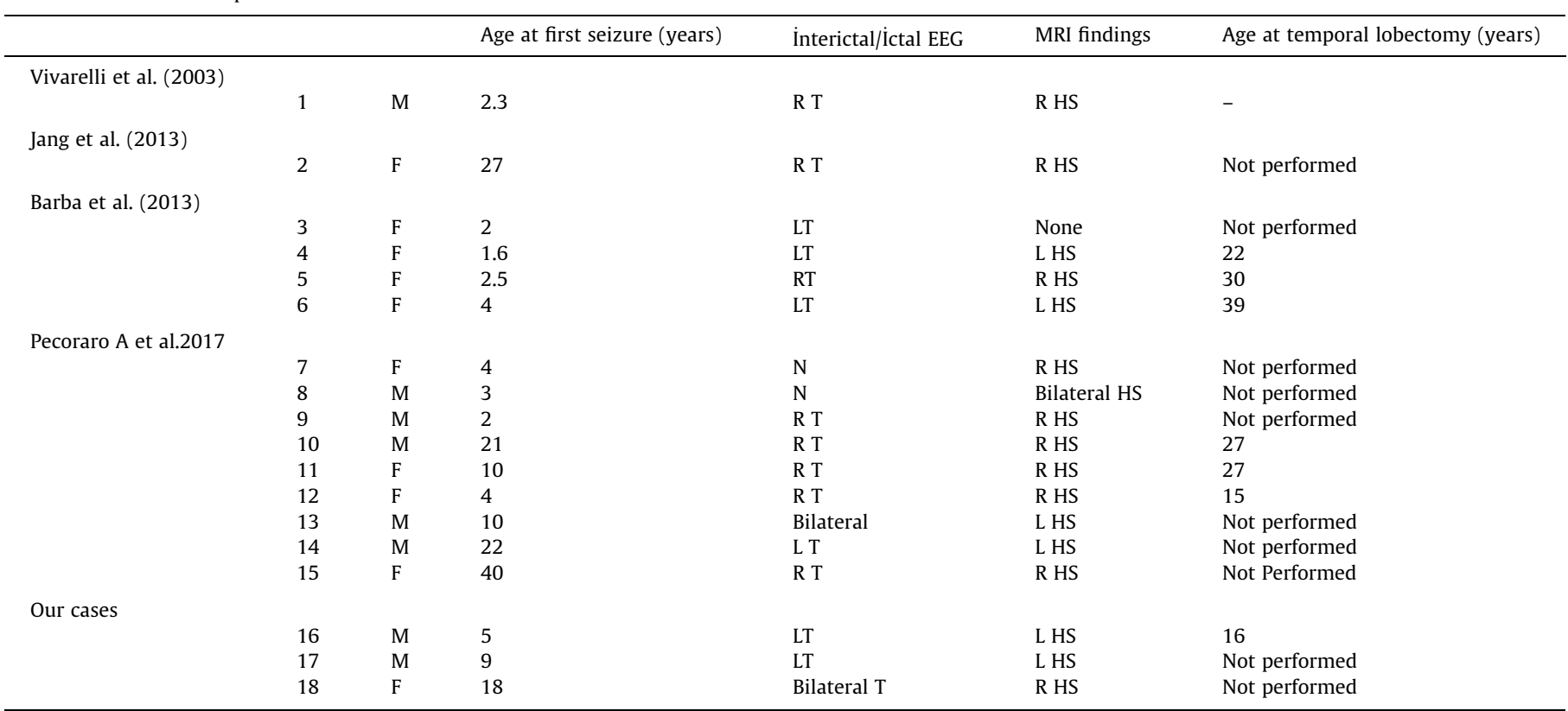

HS:Hippocampal sclerosis; T: Temporal; R: Right; L: Left; N: Normal; MRI: Magnetic Resonance Imaging. 
Carmen Barba et al., there were 12 patients with resistant epilepsy associated with NG1 and cortical development or glioneural tumour malformation on MRI. HS was identified in 4 of 12 patients. All of them were women, and 3 had left HS and 1 had right HS. 3 of 4 patients became seizure-free after temporal lobectomy [18].

Pecoraro et al. reported 9 patients with cooccurrence of NF1 and HS. Of them, 6 patients were drug-resistant. Of them, three patient received temporal lobectomy, and two patient became seizurefree. Also, two patients had dual pathology along with HS [19].

In all our 3 patients with NF1 and HS, seizures were drugresistant according to the definition of drug-resistant epilepsy of (ILAE) [20]. One patient underwent temporal lobectomy. He is seizure-free during postsurgical 5 years follow up.

Recent data obtained from NF1 patients with seizure indicate that intracranial tumours associated with NF1 are primarily responsible for the higher prevalence of epilepsy $[19,22]$. The potential pathogenic role of UBOs had been investigated and excluded. Neuroimaging performed at seizure onset revealed a new structural abnormally in $21 \%$ of cases with UBO on MRI [14].

Seizures in NF1 are often relatively easy to control with one or more conventional antiseizure drugs; surgical resection of offending lesions is sometimes pursued. Surgery has been most successful for temporal lobe gliomas [18,21]. However, if you faced the drug-resistant epilepsy you may consider the cortical malformations, tumors and HS.

As conclusion, based on the literature and our patients suggested that epilepsy in the patient with NF1 and HS is usually drug-resistant and if ictal semiology, EEG data and side of HS have concordance, temporal lobectomy is useful treatment choice for seizure control.

\section{Appendix A. Supplementary data}

Supplementary data to this article can be found online at https://doi.org/10.1016/j.jocn.2019.03.055.

\section{References}

[1] Riccardi VM, Margos VA. Characteristics of skin and tumor fibroblasts from neurofibromatosis patients. Adv Neuro 1981;29:191-8.
[2] Huson SM, Harper PS, Compston DA. Von Recklinghausen neurofibromatosis. A clinical and population study in south-east Wales. Brain 1988:111(Pt 6):1355-81.

[3] Korf BR, Carrazana E, Holmes GL. Paterns of seizures observed in association with neurofibromatosis 1. Epilepsia 1993;34(4):616-20.

[4] Friedman JM, Birch PH. Type 1 neurofibromatosis: a descriptive analysis of the disorder in 1,728 patients. Am J Med Genet 1997;70(2):138-43.

[5] Kullkantrakom K, Geller TJ. Seizures in neurofibromatosis 1. Pediatr Neurol 1998:19(5):347-50.

[6] Gill DS, Hyman SL, Steinberg A, North KN. Age-related findings on MRI in neurofibromatosis type 1. Pediatr Radiol 2006;36(10):1048-56.

[7] Ostendorf AP, Gutmann DH, Weisenberg JL. Epilepsy in individuals with neurofibromatosis type 1. Epilepsia 2013;54(10):1810-4.

[8] Runke M, Salanova V. Epilepsy due to a cortical malformation in a Neurofibromatosis type 1 patient. Seizure 2013;22(6):476-9.

[9] Szudek J, Birch P, Riccardi VM, Evans DG, Friedman JM. Associations of clinical features in neurofibromatosis 1 (NF1). Genet Epidemiol 2000;19(4):429-39.

[10] Shen MH, Harper PS, Upadhyaya M. Molecular genetics of neurofibromatosis type 1 (NF1). J Med Genet 1996;33(1):2-17.

[11] Arun D, Gutmann DH. Recent advances in neurofibromatosis type 1. Curr Opin Neurol 2004;17(2):101-5.

[12] Gill DS, Hyman SL, Steinberg A, North KN. Age-related findings on MRI in neurofibromatosis type 1. Pediatr Radiol 2006;36(10):1048-56.

[13] deBella K, Poskitt K, Szudek J, Friedman JM. Use of "unidentified bright objects" on MRI for diagnosis of neurofibromatosis 1 in children. Neurology. 2000;54 (8):1646-51.

[14] Hsieh HY, Fung HC, Wang CJ, Chin SC, Wu T. Epileptic seizures in neurofibromatosis type 1 are related to intracranial tumors but not to neurofibromatosis bright objects. Seizure 2011;20(8):606-11.

[15] Szudek J, Friedman JM. Unidentified bright objects associated with features of neurofibromatosis 1. Pediatr Neurol 2002;27(2):123-7.

[16] Vivarelli R, Grosso S, Calabrese F, Farnetani M, Di Bartolo R, Morgese G, et al. Epilepsy in neurofibromatosis 1. J Child Neurol 2003;18(5):338-42.

[17] Jang HM, Park HR, Mun JK, Hwang KJ, Kim J, Hong SC, et al. Surgical treatment of mesial temporal lobe epilepsy in a patient with neurofibromatosis type 1 . J Epilepsy Res 2013;3(1):35-8.

[18] Barba C, Jacques T, Kahane P, Polster T, Isnard J, Leijten FS, et al. Epilepsy surgery in Neurofibromatosis Type 1. Epilepsy Res 2013;105(3):384-95.

[19] Pecoraro A, Arehart E, Gallentine W, Smith E, Pizoli C, et al. Epilepsy in neurofibromatosis type 1. Epilesy Behav 2017;73:137-41.

[20] Kwan P, Arzimanoglou A, Berg AT, Allen Hauser W, Mathem G, Moshe SL, et al. Definition of drug resistant epilepsy: consensus proposal by the ad hoc Task Force of the ILAE Commission on Therapeutic Strategies. Epilepsia 2010;51 (6):1069-77.

[21] Gales J, Pravson RA. Hippocampal sclerosis and associated focal cortical dysplasia-related epilepsy in neurofibromatosis tpe I. J Clin Neurosci 2017:37:15-9.

[22] Stafstrom CE, Staedtke V, Comi AM. Epilepsy mechanisms in neurocutaneous disorders: tuberous sclerosis complex, neurofibromatosis type 1, and SturgeWeber syndrome. Front Neurol 2017;8:87. 